\title{
Forced Vibration for Electromechanical Integrated Toroidal Drive
}

\author{
Lizhong Xu, Xiuhong Hao and Hongbin Wang \\ Mechanical Engineering Institute, Yanshan University, Qinbuangdao 066004, China
}

(Received 26 September 2005; accepted 18 July 2006)

In this paper, the forced vibration of the drive system is investigated. The mesh stiffness fluctuations caused by changes of the mesh tooth pair number and the fluctuations of the polar mass moment of inertia caused by the residual unbalance in the rotor are considered. For the drive system with fluctuations of the time-varying stiffness and polar mass moment of inertia, the time-dependent coefficient dynamic equations are required. Generally, the dynamic equations can be simplified as the constant coefficient dynamic equations by transforming the fluctuations of the time-varying stiffness into equivalent exciting forces. The forced vibration of the drive system to the mesh stiffness excitation is presented by use of the constant coefficient dynamic equations. The influences of the system parameters on the forced vibration are also discussed. Under certain conditions, unstable vibrations will occur and the time-dependent coefficient dynamic equations are used to obtain the stable conditions of the system vibrations under the mesh stiffness disturbance and the unbalance disturbance in the rotor.

\section{INTRODUCTION}

The toroidal drive can transmit a large torque in a very small size unit and is suitable for technical applications such as aviation and space flight. ${ }^{1-7}$ As more and more electrical and control techniques are utilised in the mechanical engineering field, generalised composite drives are becoming used frequently. The electromagnetic harmonic drive ${ }^{8}$ and the piezoelectric harmonic drive ${ }^{9}$ are active drives in which the meshing forces between the flexible gear and the rigid gear are controlled by electromagnetic force or the piezoelectric, and drive and power are integrated.

On the basis of research on toroidal drives, ${ }^{10,11}$ the authors have presented a kind of active generalised composite drive, an electromechanical integrated toroidal drive, without contact. The drive is a new concept of the generalised composite drive. In the toroidal drive, power and control are integrated.

The drive consists of four basic elements as shown in Fig. 1: (a) the central worm; (b) radically positioned planets; (c) a toroidal shaped stator; and (d) a rotor, which forms the central output shaft upon which the planets are mounted. The central worm is fixed and coils are mounted in helical grooves of its surface. The planets have permanent magnets instead of teeth. The $N$ and $S$ polar permanent magnets are mounted alternately on a planet. The stator has helical permanent magnets instead of helical teeth. In the same manner as planet, the $N$ and $S$ polar helical permanent magnets are mounted alternately on the stator. If a specific relation among drive parameters is realised, the $N$ pole of one element will correspond to the $S$ pole of the other one. When the alternate current is connected to the coils of the worm, a toroidal circular field is formed. It drives several planets to rotate about their own axes. By means of magnetic forces between the teeth of the planet and stator, the rotor is driven to rotate about its own axis. Thus, a power of low speed and large torque is output.

Compared with the toroidal drive, the new drive is easy to produce. It is without wear and does not need lubrication. It can be substituted for a servo system to simplify the structure of existing electromechanical systems. Besides the abovementioned fields that require compactness, the drive can be used with robots and in other fields that require accurate control.

In this paper, the forced vibration of the drive system is investigated. The mesh stiffness fluctuations caused by changes of the mesh tooth pair number and the fluctuations of the polar mass moment of inertia caused by the residual unbalance in the rotor are considered. For the drive system, with the fluctuations of the time-varying stiffness and polar mass moment of inertia, the time-dependent coefficient dynamic equations are required. Generally, the dynamic equations can be simplified as the constant coefficient dynamic equations by transforming the fluctuations of the timevarying stiffness into equivalent exciting forces. By the constant coefficient dynamic equations, the forced vibrations of the drive system to the mesh stiffness excitation are presented. The influences of the system parameters on the forced vibrations are also discussed. Under certain conditions, unstable vibrations will occur and the time-dependent coefficient dynamic equations are used to obtain the stable conditions of the system vibrations under the mesh stiffness disturbance and the unbalance disturbance in the rotor. The results are useful in design and manufacture of the drive and can be used to design parameters of the drive and remove undesirable dynamic behaviour.

\section{ELECTROMECHANICAL COUPLED DYNAMIC EQUATIONS}

The dynamic model of the mechanical system for the drive is shown in Fig. 2. The dynamic model shown in Fig. 2 allows the rotor and each planet to rotate about their own rotating axes, and allows each planet to translate in $x_{i}$ and $z_{i}$ directions. For the sake of convenience, the rotations are replaced by their corresponding translational mesh displacements as $u_{j}=r_{j} \theta_{j}, j=1, \ldots, m, r$. Here, $\theta_{j}$ is the rotation of planet or rotor, $r_{j}$ is the base circle radius for planet and sta- 